\title{
A phase II, single-centre trial of neoadjuvant toripalimab plus chemotherapy in locally advanced esophageal squamous cell carcinoma
}

\author{
Wenqun Xing ${ }^{1 *}$, Lingdi Zhao ${ }^{2 \#}$, Xiaomin Fu' ${ }^{2}$, Guanghui Liang ${ }^{1}$, Yong Zhang ${ }^{2}$, Dongfeng Yuan ${ }^{1}$, \\ Zhenxuan $\mathrm{Li}^{1}$, Quanli Gao ${ }^{2}$, Yan Zheng ${ }^{1}$; written on Henan Cancer Hospital Thoracic Oncology Group \\ (HCHTOG)
}

\begin{abstract}
${ }^{1}$ Department of Thoracic Surgery, The Affiliated Cancer Hospital of Zhengzhou University, Henan Cancer Hospital, Zhengzhou, China; ${ }^{2}$ Department of Immunotherapy, The Affiliated Cancer Hospital of Zhengzhou University, Henan Cancer Hospital, Zhengzhou, China Contributions: (I) Conception and design: Q Gao, W Xing, Y Zheng; (II) Administrative support: Q Gao, W Xing, Y Zheng; (III) Provision of study materials or patients: L Zhao, X Fu, G Liang, Y Zhang, D Yuan, Z Li; (IV) Collection and assembly of data: L Zhao, X Fu, G Liang, Y Zhang, D Yuan, Z Li,; (V) Data analysis and interpretation: Q Gao, W Xing, Y Zheng; (VI) Manuscript writing: All authors; (VII) Final approval of manuscript: All authors.

\#These authors contributed equally to this article.

Correspondence to: Quanli Gao, MD, PhD. Department of Immunotherapy, The Affiliated Cancer Hospital of Zhengzhou University, Henan Cancer Hospital, Zhengzhou 450008, China. Email: gaoquanli2015@126.com; Yan Zheng, MD, PhD. Department of Thoracic Surgery, The Affiliated Cancer Hospital of Zhengzhou University, Henan Cancer Hospital, Zhengzhou 450008, China. Email: sunnyzheng1@126.com.
\end{abstract}

Background: Esophageal squamous cell carcinoma (ESCC) remains a challenging malignancy with poor prognosis and limited therapeutic methods. However, recent clinical trials of immune checkpoint inhibitors (ICIs) have shown promising results in the treatment of lethal malignancies. The second-line treatment of late-stage ESCC was approved based on the results of KEYNOTE-180, KEYNOTE-181 and ATTRACTION-1, ATTRACTION-3. Combining ICIs with chemotherapy in neoadjuvant therapy may benefit patients with locally advanced, resectable ESCC.

Methods: A two-arm phase II trial was launched in July 2019 in Henan Cancer Hospital. The primary outcome measure will be completed within 21 months. The pathological complete response (pCR) rate is the primary endpoint, and the secondary endpoints include overall survival (OS), disease-free survival (DFS), the toxicities of the neoadjuvant toripalimab plus chemotherapy, the relationship between combined positivity score (CPS) of specimen and the treatment response, the relationship between lymphocyte infiltration and the treatment response, the progression-free survival (PFS) rate, and adverse events (AEs). It was assumed that the pCR rate of this trial might be $25 \%$. Therefore, the 30 enrolled patients could reject the hypothesis at $75 \%(\alpha=0.1)$.

Discussion: The study will determine the safety and efficacy of neoadjuvant immunochemotherapy for ESCC and provide enough evidence for phase III clinical trials.

Trial registration: Clinical Trials.gov, NCT03985670, Registered: October 24, 2019, https://register. clinicaltrials.gov/prs/app/action/SelectProtocol?sid=S0008Z9D\&selectaction=Edit\&uid=U0002MIY\&ts=2\& cx=-i71o4q. Registry name: "Teripalimab Plus Chemotherapy in Local Advanced Esophageal Cancer".

Keywords: Esophageal cancer; neoadjuvant immunochemotherapy; clinical trial; phase II

Submitted Jun 13, 2020. Accepted for publication Sep 23, 2020.

doi: $10.21037 /$ jtd-20-2198

View this article at: http://dx.doi.org/10.21037/jtd-20-2198 


\section{Introduction}

Esophageal cancer was diagnosed in 572,034 patients globally in 2018 (1). Esophageal squamous cell carcinoma (ESCC) is more likely to occur in Africa (2) and Eastern Asia (3). China contributed $53 \%$ of the global ESCC (4). ESCC is notable for its regional distribution (5). Even within China, Henan Province has the highest incidence of ESCC, with an estimated 36,840 new cases every year (6). In the past 30 years, a series of multi-institutional clinical trials have demonstrated that neoadjuvant chemotherapy and chemoradiotherapy have improved the 5 -year OS of ESCC $(7,8)$. The NEORES has showed no survival difference between neoadjuvant chemotherapy (NAC) and Neoadjuvant chemoradiotherapy (NACR) (9). Although there is still debate regarding whether chemotherapy or chemoradiotherapy is more effective, the prize may be swiftly won by immunotherapy with immune checkpoint inhibitors (ICIs) that target the programmed cell death receptor 1 (PD-1)/programmed cell death ligand 1 (PD-L1) pathway. The use of immunotherapy for the treatment of malignancies has been expanding rapidly since the release of ipilimumab in 2011 (10). This promising avenue for ESCC treatment has been the subject of ongoing research in recent years (11).

Remarkable progress has been made recently in immunotherapies for the treatment of ESCC. The antiPD-1 monoclonal antibodies (mAbs) pembrolizumab and nivolumab were approved by the FDA for the second-line treatment of advanced ESCC patients (10).

Thus, clinical therapy combining NAC and the antiPD-1 antibody toripalimab might produce a better anticancer effect and improve survival. The safety profiles of immunochemotherapy agents have already been established. Taking all these factors into account, the current exploratory trial was launched. We present the following article in accordance with the SPIRIT reporting checklist (available at http://dx.doi.org/10.21037/jtd-20-2198).

\section{Methods}

\section{Statement of ethics approval}

The ethics committee and institutional review board of Henan Cancer Hospital approved this study and the ethical approach (ID: 2019205). All the participants will sign informed consent forms. The trial will be performed following the ethical principles of the Declaration of Helsinki (12) (as revised in 2013).

\section{Study design and setting}

This is a phase II, single-centre, open label clinical trial. Eligible patients are randomized to arm A (paclitaxel 150 $175 \mathrm{mg} / \mathrm{m}^{2} \mathrm{~d} 1+$ cisplatin $70-75 \mathrm{mg} / \mathrm{m}^{2} \mathrm{~d} 1+$ toripalimab $240 \mathrm{mg} \mathrm{d} 1$ ) and arm B (paclitaxel 150-175 mg/m $\mathrm{m}^{2} \mathrm{~d} 1+$ cisplatin $70-75 \mathrm{mg} / \mathrm{m}^{2} \mathrm{~d} 1$ + toripalimab $240 \mathrm{mg} \mathrm{d} 3$ ) and then undergo surgery with a 1:1 allocation ratio (Figure 1). The aim of this trial is to investigate the pCR rate and safety of the neoadjuvant toripalimab plus chemotherapy for resectable locally advanced ESCC.

\section{Study endpoint}

The primary outcome is the pathological complete response (pCR) rate, defined as the proportion of patients who achieved pCR. The results will be based on the pathologic diagnosis of removed specimens after surgery.

The secondary outcomes include overall survival (OS), disease-free survival (DFS), the toxicities of neoadjuvant toripalimab plus chemotherapy, the relationship between combined positivity score (CPS) of specimen and the treatment response, the relationship between lymphocyte infiltration and the treatment response, and adverse events [AEs, National Cancer Institute Common Terminology Criteria for Adverse Event, Version 4.0 (CTC AE4.0)].

The time frame for reporting AEs in each patient will be up to 3 months after the last dose of neoadjuvant treatment. We defined OS as the number of days from randomization to death for any reason. PFS is defined as the number of days from randomization to progression or death.

\section{Participants}

In the Affiliated Cancer Hospital of Zhengzhou University/ Henan Cancer Hospital, the first diagnosed ESCC patients will receive physical examination, esophageal endoscopic ultrasound, contrast-enhanced thoracic CT scan, oesophagography, abdominal ultrasound, cervical colour ultrasound, brain MRI and emission computed tomography (ECT). If the patient agrees, positron emission tomographyCT (PET/CT) will be used rather than cervical colour ultrasound, chest CT, abdominal ultrasound and ECT. All patients will be given a pretreatment staging based on the 8th UICC TNM system (13). The multidisciplinary team (MDT) of the Thoracic Oncology Group will discuss all ESCC patients before any treatment, and suitable patients will be selected. Written informed consent will be obtained 


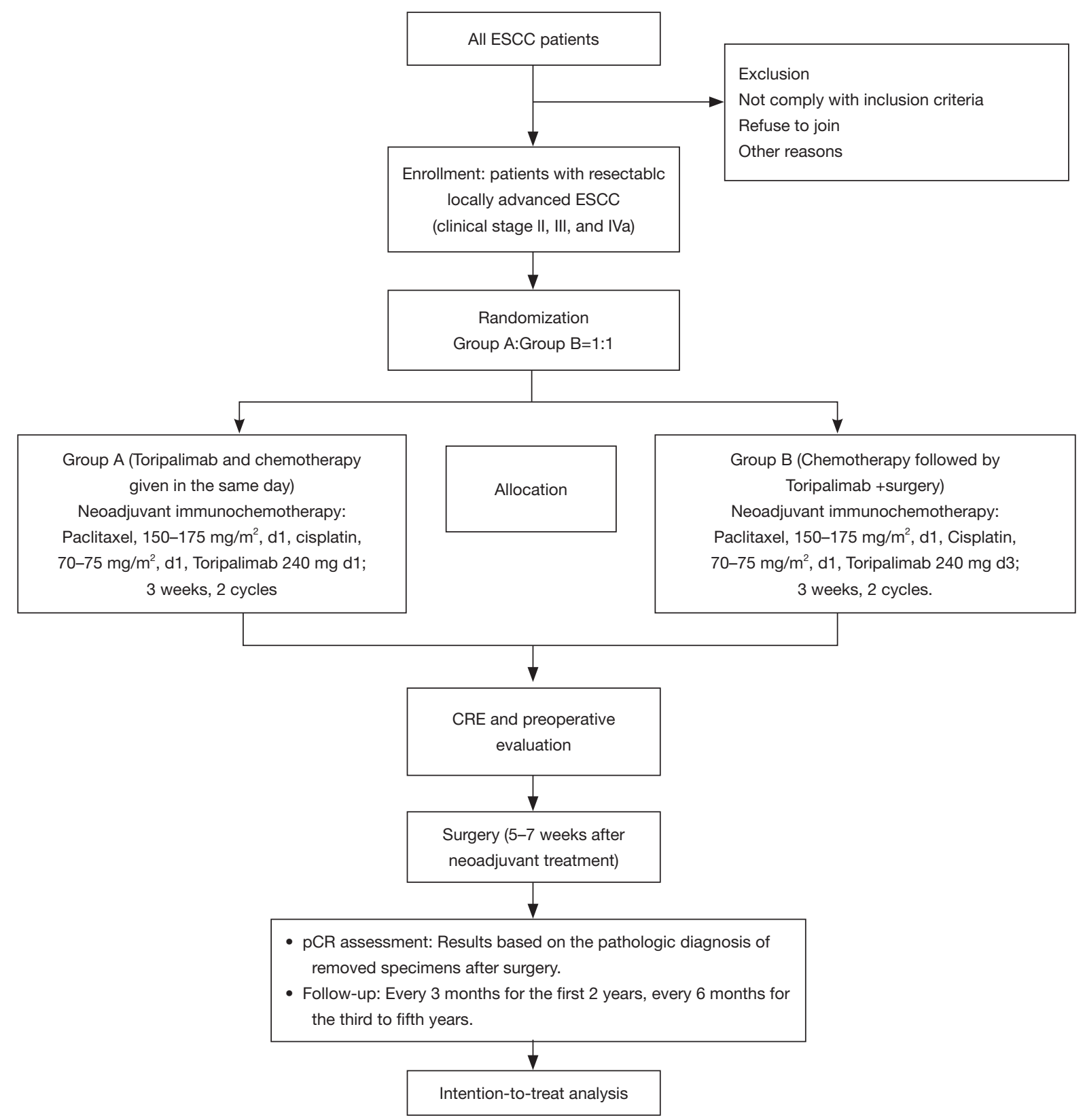

Figure 1 Flowchart of the phase II study. ESCC, oesophageal squamous cell carcinoma; CRE, clinical response evaluation; pCR, pathologic complete response.

from all patients prior to recruitment for the trial.

Inclusion criteria:

(I) Histologic diagnosis of thoracic esophageal squamous cell carcinoma.

(II) No distant metastasis. R0 resection is expected by the thoracic oncologic MDT.

(III) ECOG score 0-1.

(IV) Clinical stage II, III, or IVa according to the
American Joint Committee on Cancer (AJCC) 8.0.

(V) Life expectancy more than 3 months.

(VI) Sufficient organ function.

(VII) Normal blood pressure. For patients with hypertension, blood pressure should be controlled at a normal level by antihypertension medication.

(VIII) Fasting blood sugar $\leq 8 \mathrm{mmol} / \mathrm{L}$ for diabetic patients. 
(IX) No other serious diseases (such as autoimmune diseases, immunodeficiencies, organ transplants) that conflict with this study.

(X) No history of other malignant tumours.

(XI) Women must have a negative blood test for pregnancy within 7 days and use appropriate contraceptive measures during the trial period and within 6 months after the trial.

(XII) The patient agrees to participate in the clinical study and signs the informed consent form.

Exclusion criteria:

(I) Serious infectious disease within 4 weeks before enrolment.

(II) Bronchial asthma requiring intermittent use of bronchodilators or medical interventions.

(III) Usage of immunosuppressants before enrolment: $\geq 10 \mathrm{mg} /$ day oral prednisone for more than 2 weeks.

(IV) Clinically significant cardiovascular and cerebrovascular diseases, including but not limited to severe acute myocardial infarction, unstable or severe angina, coronary artery bypass surgery, congestive heart failure, incidents requiring medical intervention within the first 6 months of enrolment, arrhythmia, and left ventricular ejection fraction $<50 \%$.

(V) Severe allergies.

(VI) Severe mental disorder.

(VII) Abnormal blood coagulation function (PT $>16 \mathrm{~s}$, APTT $>53 \mathrm{~s}$, TT $>21 \mathrm{~s}$, Fib $<1.5 \mathrm{~g} / \mathrm{L}$ ), bleeding tendency or receiving thrombolysis or anticoagulant therapy.

(VIII) Previous or current pulmonary fibrosis, interstitial pneumonia, pneumoconiosis, radiation pneumonitis, severe lung damage, etc.

(IX) Other situations evaluated by investigators as not suitable for enrolment.

\section{Masking and randomization}

A clinical research nurse takes responsibility for randomization. After confirmation of the recruitment criteria, the patients are randomized to arm A or arm B.

This study is unmasked. The randomization numbers are generated by a computer. All the randomized results are sealed into envelopes. Patients are randomly allocated (1:1). All the personal data was collected by a research nurse with CRF. All the data would be double checked.

\section{Treatment regimens}

Arm A (toripalimab and chemotherapy given on the same day)

Paclitaxel, $150-175 \mathrm{mg} / \mathrm{m}^{2}$, d1, Cisplatin, $70-75 \mathrm{mg} / \mathrm{m}^{2}$, d1, toripalimab $240 \mathrm{mg} \mathrm{d} 1 ; 3$ weeks, 2 cycles.

\section{Arm B (chemotherapy followed by toripalimab)}

Paclitaxel, 150-175 mg/m², d1, Cisplatin, 70-75 mg/m², d1, toripalimab $240 \mathrm{mg} \mathrm{d} 3 ; 3$ weeks, 2 cycles.

\section{Surgery}

Three to seven weeks after neoadjuvant treatment, surgery will be performed. Thoracoscopic oesophagectomy, open thoracotomy oesophagectomy and hybrid approaches with a total 2-field lymphadenectomy will be performed.

\section{Follow-up}

The primary endpoints will be analysed 2 months after the surgical treatment of the last recruited patient. The surveillance examinations include chest CT scans and abdominal and cervical colour Doppler ultrasonography. All the tests will be performed in the outpatient department. Visits will occur every 3 months for the first 2 years and every 6 months for the third to fifth years.

\section{Statistical analysis}

This trial is designed to explore the effectiveness (pCR rate) and safety (AE and SAE) of neoadjuvant immunochemotherapy followed by oesophagectomy with complete two-field lymphadenectomy. The pCR rate for NAC was about $10 \%$. It was assumed that the pCR rate of this trial might be $25 \%$. Therefore, the 30 enrolled patients could reject the hypothesis at $75 \%(\alpha=0.1)$. As an exploratory study, the sample size was 30 totally. SPSS 23.0 software for Windows (SPSS, Chicago, IL, USA) will be adopted for the statistical analysis. The intent to treatment (ITT), Per Protocol Set (PPS) and safety set (SS) will be analysed and reported.

\section{Monitoring}

The good clinical practice (GCP) board and IRB of the Affiliated Cancer Hospital of Zhengzhou University/Henan Cancer Hospital will evaluate patient safety, study progress and data integrity. The GCP will review the trial data 
every 6 months. It is independent and without competing interests. The principal investigator (PI) takes responsibility for trial design and performance. The PIs will make the final trial database available.

\section{Participating institutions}

Henan Cancer Hospital/The Affiliated Cancer Hospital of ZhengZhou University. Principal Investigator: Quanli Gao, M.D.\& Ph.D., Wenqun Xing, M.D.

\section{Funding, registration and current status}

This study was funded by Henan Cancer Hospital (2019205) (Dr. Quanli Gao, gaoquanli2015@126.com). This is a The TopAlliance offered the Toripalimab for free for this study. This is an investigator initiated trial (IIT).

This study was registered before recruitment at ClinicalTrials.gov in October 2019 (registration number: NCT03985670). The protocol date and version identifier is version 1 (March 25, 2019). Our study began recruitment in October 2019. The trial status is patient recruitment.

\section{Discussion}

ESCC was reported to be the fifth leading cause of cancerrelated death each year and the ninth most common cancer worldwide in 2018 (1) by the International Agency for Cancer Research (IARC). Surgery remains the mainstay of treatment for resectable ESCC. Multidisciplinary management has been proven to provide survival benefits (14). Because of the regional distribution of ESCC, some areas have adopted NAC as standard treatment, and others have adopted NACR. However, the survival benefit of these treatments is still limited. In 2011, ipilimumab was approved for cancer treatment. In 2019, positive results have been reported for ICIs as second-line ESCC treatment in many phase III RCTs. More than 20 indications and approximately 15 cancer types were proven to respond to anti-PD-1/PD-L1 antibodies (15).

If ICIs are adopted early in the neotreatment setting for resectable ESCC, they may provide more survival benefits. To date, even after neoadjuvant chemotherapy (16), approximately $50 \%$ of ESCC patients relapse within 1 year after surgery. If the patient does not achieve a complete pathological response, the recurrence rate is even higher (17). We anticipated that neoadjuvant immunotherapy may establish a new therapeutic paradigm in ESCC.

Immunotherapy is now under investigation in locally resectable disease. In basic research, PD-L1 in esophageal adenocarcinoma may be upregulated by pretreatment chemoradiation (18). Kelly et al. reported a phase Ib study evaluating nivolumab plus neoadjuvant chemoradiation in esophageal/GEJ cancer (19). Unexpected AEs were not observed. Five out of 15 resected ESCC patients achieved pCR. NCT03604991, NCT04006041 and NCT03288350 all explored the combination of ICIs and neoadjuvant chemoradiotherapy. The only phase III clinical trial in the neoadjuvant setting is NCT03604991, which tested nivolumab alone or with ipilimumab and neoadjuvant chemotherapy in esophageal and GEJ adenocarcinoma. The pCR rate was the primary endpoint, and the second endpoint was DFS. In Asian countries, especially in Japan, neoadjuvant chemotherapy is the standard treatment. The surgical methods and the pathological type are similar in China and Japan. Keynotes 180, 181 and ATTRACTION-1, 3 have already shown the successful combination of ICIs and chemotherapy. If we want to obtain a better cost and benefit rate, neoadjuvant immunochemotherapy should be evaluated.

There has been limited data to explore the sequence of ICIs and chemotherapy. Huang et al. reported that the pretreated tumour burden was related to the reinvigoration of phenotype CD8 $\mathrm{T}$ cells after pembrolizumab treatment and was correlated with the tumour response $(20,21)$. Other studies have suggested the opposite direction: they proposed that ICIs before chemotherapy may allow a better response because of the preservation of immune cells. Otherwise, chemotherapy may lead to immunosuppression and myelosuppression, limiting the effects of ICIs (22). To obtain primary results on the different sequences of chemotherapy and ICI combinations, we designed two study arms.

For resectable locally advanced ESCC patients, some trials of ICI-containing preoperative investigational chemoradiotherapies are reported (NCT02844075, NCT03490292, NCT03044613) and a trial of nivolumabcontaining preoperative chemotherapy is ongoing (NCT03914443). A prominent characteristic of ESCC is its regional distribution. Henan Province has the highest incidence of ESCC in China. Clinical trials, especially largescale phase III clinical studies, may be easier to complete in Henan Province (23). As the largest cancer centre in Henan Province, we should launch phase II and phase III studies to answer the cutting-edge questions. 


\section{Conclusions}

ICIs have shown strong evidence of anticancer effects in advanced ESCC. Herein, there is a strong reason to explore the effects of ICIs in combination with chemotherapy in neoadjuvant treatment for localized ESCC. Therefore, this phase II clinical study was designed and launched to evaluate the efficacy and safety of toripalimab for resectable ESCC. Hopefully, the phase II study will elucidate the role of the neoadjuvant approach (toripalimab plus chemotherapy) for ESCC and provide enough evidence for phase III clinical trials.

This paper has been prepared in line with standard protocol items Recommendations for Interventional Trials (SPIRIT) (24).

\section{Acknowledgments}

Funding: This study was funded by Henan Cancer Hospital (2019205).

\section{Footnote}

Reporting Checklist: The authors have completed the SPIRIT reporting checklist. Available at http://dx.doi.org/10.21037/ jtd-20-2198.

Conflicts of Interest: All authors have completed the ICMJE uniform disclosure form (available at http://dx.doi. org/10.21037/jtd-20-2198). The authors have no conflicts of interest to declare.

Ethical Statement: The authors are accountable for all aspects of the work in ensuring that questions related to the accuracy or integrity of any part of the work are appropriately investigated and resolved. The ethics committee and institutional review board of Henan Cancer Hospital approved this study and the ethical approach (ID: 2019205). All the participants will sign informed consent forms. The trial will be performed following the ethical principles of the Declaration of Helsinki (as revised in 2013).

Open Access Statement: This is an Open Access article distributed in accordance with the Creative Commons Attribution-NonCommercial-NoDerivs 4.0 International License (CC BY-NC-ND 4.0), which permits the noncommercial replication and distribution of the article with the strict proviso that no changes or edits are made and the original work is properly cited (including links to both the formal publication through the relevant DOI and the license). See: https://creativecommons.org/licenses/by-nc-nd/4.0/.

\section{References}

1. Bray F, Ferlay J, Soerjomataram I, et al. Global cancer statistics 2018: GLOBOCAN estimates of incidence and mortality worldwide for 36 cancers in 185 countries. CA Cancer J Clin 2018;68:394-424.

2. Somdyala NI, Bradshaw D, Gelderblom WC, et al. Cancer incidence in a rural population of South Africa, 1998-2002. Int J Cancer 2010;127:2420-9.

3. Lin Y, Totsuka Y, He Y, et al. Epidemiology of esophageal cancer in Japan and China. J Epidemiol 2013;23:233-42.

4. Siegel R, Desantis C, Jemal A. Colorectal cancer statistics, 2014. CA Cancer J Clin 2014;64:104-17.

5. Arnold M, Soerjomataram I, Ferlay J, et al. Global incidence of oesophageal cancer by histological subtype in 2012. Gut 2015;64:381-7.

6. Chen $\mathrm{W}$, Zheng R, Zuo T, et al. National cancer incidence and mortality in China, 2012. Chin J Cancer Res 2016;28:1-11.

7. Zheng Y, Li Y, Liu X, et al. Reevaluation of Neoadjuvant Chemotherapy for Esophageal Squamous Cell Carcinoma: A Meta-Analysis of Randomized Controlled Trials Over the Past 20 Years. Medicine (Baltimore) 2015;94:e1102.

8. van Heijl M, van Lanschot JJ, Koppert LB, et al. Neoadjuvant chemoradiation followed by surgery versus surgery alone for patients with adenocarcinoma or squamous cell carcinoma of the esophagus (CROSS). BMC Surg 2008;8:21.

9. von Döbeln GA, Klevebro F, Jacobsen AB, et al. Neoadjuvant chemotherapy versus neoadjuvant chemoradiotherapy for cancer of the esophagus or gastroesophageal junction: long-term results of a randomized clinical trial. Dis Esophagus 2019;32. doi: 10.1093/dote/doy078.

10. Chuang J, Chao J, Hendifar A, et al. Checkpoint inhibition in advanced gastroesophageal cancer: clinical trial data, molecular subtyping, predictive biomarkers, and the potential of combination therapies. Transl Gastroenterol Hepatol 2019;4:63.

11. Huang TX, Fu L. The immune landscape of esophageal cancer. Cancer Commun (Lond) 2019;39:79.

12. World Medical Association Inc. Declaration of Helsinki. Ethical principles for medical research involving human subjects. J Indian Med Assoc 2009;107:403-5. 
13. Rice TW, Ishwaran H, Ferguson MK, et al. Cancer of the Esophagus and Esophagogastric Junction: An Eighth Edition Staging Primer. J Thorac Oncol 2017;12:36-42.

14. Lordick F, Mariette C, Haustermans K, et al. Oesophageal cancer: ESMO Clinical Practice Guidelines for diagnosis, treatment and follow-up. Ann Oncol 2016;27:v50-7.

15. Vivaldi C, Catanese S, Massa V, et al. Immune Checkpoint Inhibitors in Esophageal Cancers: are we Finally Finding the Right Path in the Mist? Int J Mol Sci 2020;21:1658.

16. van Hagen $P$, Hulshof MC, van Lanschot JJ, et al. Preoperative chemoradiotherapy for esophageal or junctional cancer. N Engl J Med 2012;366:2074-84.

17. Kesler KA, Helft PR, Werner EA, et al. A retrospective analysis of locally advanced esophageal cancer patients treated with neoadjuvant chemoradiation therapy followed by surgery or surgery alone. Ann Thorac Surg 2005;79:1116-21.

18. Kelly RJ, Zaidi AH, Smith MA, et al. The Dynamic and Transient Immune Microenvironment in Locally Advanced Esophageal Adenocarcinoma Post Chemoradiation. Ann Surg 2018;268:992-9.

19. Kelly RJ, Smith KN, Anagnostou V, et al. Neoadjuvant nivolumab plus concurrent chemoradiation in stage II/III

Cite this article as: Xing W, Zhao L, Fu X, Liang G, Zhang Y, Yuan D, Li Z, Gao Q, Zheng Y; written on Henan Cancer Hospital Thoracic Oncology Group (HCHTOG). A phase II, single-centre trial of neoadjuvant toripalimab plus chemotherapy in locally advanced esophageal squamous cell carcinoma. J Thorac Dis 2020;12(11):6861-6867. doi: 10.21037/ jtd-20-2198 esophageal/gastroesophageal junction cancer. J Clin Oncol 2019;37:142.

20. Huang AC, Postow MA, Orlowski RJ, et al. T-cell invigoration to tumour burden ratio associated with antiPD-1 response. Nature 2017;545:60-5.

21. Yamasaki M, Funaishi K, Kawamoto K, et al. Platinumdoublet chemotherapy followed by pembrolizumab therapy for lung cancer with lymphangitis carcinomatosa mimicking interstitial pneumonitis: A case report.

Medicine (Baltimore) 2019;98:e16834.

22. Ramchandren R, Domingo-Domenech E, Rueda A, et al. Nivolumab for Newly Diagnosed Advanced-Stage Classic Hodgkin Lymphoma: Safety and Efficacy in the Phase II CheckMate 205 Study. J Clin Oncol 2019;37:1997-2007.

23. Zheng Y, Li Y, Liu X, et al. A phase III, multicenter randomized controlled trial of neo-adjuvant chemotherapy paclitaxel plus cisplatin versus surgery alone for stage IIAIIIB esophageal squamous cell carcinoma. J Thorac Dis 2017;9:200-4.

24. Moher D, Altman DG, Schulz KF, et al. SPIRIT (Standard Protocol Items: Recommendations for Interventional Trials). John Wiley \& Sons, Ltd, 2014:56-67. 